https://artnodes.uoc.edu

\title{
Cultura de la participación cívica: movilización transmedia y hacktivismo en comunidades de práctica (casos de fans y makers)
}

\author{
José Manuel Corona-Rodríguez \\ Instituto Tecnológico y de Estudios Superiores de Monterrey
}

Fecha de presentación: octubre de 2021

Fecha de aceptación: enero de 2022

Fecha de publicación: enero de 2022

\section{Cita recomendada}

Corona-Rodríguez, José Manuel. 2022. «Cultura de la participación cívica: movilización transmedia y hacktivismo en comunidades de práctica (casos de fans y makers)». En: Garcés, Marina (coord.). «Ecología de la imaginación». Artnodes, no. 29. UOC. [Fecha de consulta: dd/mm/aa]. https://doi. org/10.7238/artnodes.v0i29.393059

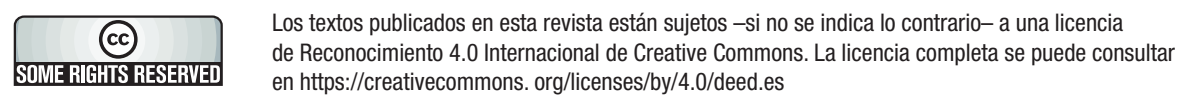

\section{Resumen}

Este artículo presenta los resultados de una investigación etnográfica sobre las prácticas de participación (orientadas a causas cívicas) realizadas por dos comunidades de práctica en el contexto de la cultura participativa. Se propone el concepto de cultura de la participación cívica como una apuesta por evidenciar las prácticas de participación relacionadas con temas y causas cívicas, a partir de relatos y discursos mediáticos ficcionales y basados en el movimiento maker. La metodología desarrolla una estrategia que recupera las formas de agencia comunicativa presencial y virtual de las personas integrantes de dos comunidades de práctica. Se realiza una propuesta de categorización de las prácticas observadas a partir de la movilización transmedia que incorpora referentes de la cultura popular al involucramiento cívico de los participantes, y de una forma de hacktivismo fan caracterizado por el uso creativo y alternativo de la tecnología digital y analógica para la resolución de problemas sociales. 


\title{
artnodes
}

https://artnodes.uoc.edu

Cultura de la participación cívica: movilización transmedia y hacktivismo en comunidades de práctica (casos de fans y makers)

Palabras clave

cultura de la participación; movilización transmedia; hacktivismo; fans; comunidades

\author{
Culture of civic participation: transmedia mobilization and hacktivism in communities of practice \\ (makers and fans cases)
}

\begin{abstract}
This article presents the results of an ethnographic study on the participatory practices (civic cause-orientated) carried out by two communities of practice in the context of participatory culture. The concept of culture of civic participation is proposed as a way to demonstrate the practices of participation related to civic issues and causes, based on fictional media narratives and discourses and on the maker movement. The methodology develops a strategy that recovers the forms of face-to-face and virtual communicative agency of the members of two communities of practice. A proposal is made to categorize the observed practices based on the transmedia mobilization that incorporates referents of popular culture to the civic involvement of the participants, and a form of fan hacktivism characterized by the creative and alternative use of digital and analogue technology to solve social problems.
\end{abstract}

\section{Keywords}

participatory culture; transmedia mobilization; hacktivism; fans; communities

\section{Introducción}

En este texto se documenta una investigación sobre dos comunidades de práctica (una de fans y otra de makers²), a partir del análisis de sus formas de comunicación y organización relativas a problemas sociales con una orientación cívica, para lo cual se despliegan y proponen las categorías de análisis: movilización transmedia y hacktivismo de fans.

Como parte de la argumentación, se describen y analizan las prácticas observadas de las comunidades y cómo es que estas se vinculan a formas de participación relativas a causas cívicas. Desde la perspectiva de la movilización transmedia (Costanza-Chock 2010) se trabaja sobre el involucramiento de un grupo de personas pertenecientes a un movimiento social en la realización de mensajes y productos comunicativos que promuevan las causas, objetivos o posturas de aquello que da sentido y sustento a las prácticas compartidas a partir de la cultura popular o como resultado de la interacción con relatos y narrativas mediáticas.

Desde el hacktivismo (Avogadro 2014, Coleman 2014) se busca evidenciar el desarrollo de un tipo de activismo basado en el uso de la tecnología y/o en el cuestionamiento crítico de los sistemas hegemónicos técnicos. Y se propone la existencia de un tipo de hacktivismo de fans entendido como una modalidad alternativa de la movilización transmedia, y caracterizada por el uso y apropiación creativo de la tecnología (tanto digital como analógica) para la resolución, visibilización 0 intermediación de problemas sociales concretos.

El contexto general en el que se sitúa este texto problematiza las constantes transformaciones tecnológicas y culturales que han influido en la evolución de la comunicación y la educación ${ }^{3}$ como dos disciplinas que se han transformado significativamente en los últimos años. ${ }^{4}$

\section{Culturas y prácticas de participación}

Cultura de la participación (Aparici y 0suna-Acedo 2013) o cultura participativa es un concepto popularizado en los últimos años en los estudios de la comunicación, especialmente en los debates sobre las transformaciones en los tipos de producción y consumo realizado por las personas luego de la llegada y el establecimiento de la conver-

1. El concepto fans se entiende como una profunda implicación hacia a una historia, producto, contenido o sistema de valores, etc. El vínculo de los sujetos con el objeto de su afición está basado en el interés personal y compartido para seguir conociendo y aprendiendo de aquello que les interesa.

2. Se desprende del movimiento maker, que se refiere a un conjunto de acciones y actitudes basadas en la transformación, fabricación y creación de objetos materiales, a través de la desintermediación y el uso de tecnologías y herramientas digitales 0 analógicas.

3. Este texto es resultado del trabajo realizado por el autor en el marco de su investigación doctoral, y que se extendió hasta febrero de 2020.

4. El marco general de la investigación aquí reportada se puede caracterizar a partir del escenario convergente mediático y el debate sobre la capacidad de agencia del creador/productor y los consumidores frente a las múltiples experiencias comunicativas que suceden en la actualidad. 


\section{artnodes}

https://artnodes.uoc.edu

Cultura de la participación cívica: movilización transmedia y hacktivismo en comunidades de práctica (casos de fans y makers)

gencia comunicativa (Jenkins 2014), caracterizada por la integración de múltiples medios y discursos en la transformación de los roles de los medios, de las industrias comunicativas, de las personas y de las plataformas tecnológicas y el creciente uso masivo de los datos (Mejías y Couldry 2019). Popularizado mayormente por el trabajo de Henry Jenkins (2008), la cultura participativa 0 de la participación significa una transformación en las condiciones técnicas, económicas y culturales que permiten que las personas se involucren en la creación y no solo en el consumo de productos mediáticos.

Es cierto que a lo largo de por lo menos la reciente década han existido críticas diversas al concepto de la cultura participativa, dado que se ha asumido que las formas de participación ocurren independientemente de las condiciones económicas, tecnológicas o educativas de las personas que acceden, producen o consumen información. Uno de los críticos más puntuales ha sido Couldry (2011), quien sugiere que para hablar de una verdadera cultura de la participación se tendrían que considerar las diferencias en términos de la estratificación de la sociedad, y la problematización de la idea de cultura como concepto complejo que disemina el sentido y símbolos que son referencia para las personas. Otros cuestionamientos a las formas de participan se concentran en la dimensión económica que subyace a las interacciones que son posibles a través de espacios virtuales y sociodigitales; un ejemplo claro es la propuesta contra el freemium (Evans 2015, Hamari, Hanner y Koivisto 2020), el cual consiste en un modelo de negocios que se sustenta en el ofrecimiento de servicios básicos gratuitos, que luego se traducen en cobros por servicios avanzados o exclusivos.

Una definición apropiada de la cultura de la participación sería aquella que considera no una sola cultura sino muchas formas culturales en las que se manifiesta y ocurre la agencia individual, siempre considerando las condiciones externas estructurales de las industrias, el mercado y el rol de los Estados. Desde esta perspectiva, y luego de los resultados de esta investigación, proponemos la existencia de una cultura de la participación cívica, orientada en función de prácticas comunicativas que buscan influir en la resolución de una problemática social.

Basándonos en la propuesta de Jenkins (2009), asumimos la cultura participativa a partir de las siguientes características:

a) La existencia de condiciones para la expresión y el compromiso social.

b) Las circunstancias socioafectivas para crear y compartir las producciones.

c) La coexistencia de personas dispuestas a compartir sus conocimientos y experiencias para que otros menos experimentados logren la integración en los grupos sociales.

d) Miembros de comunidades que se asuman como relevantes en un grupo y que puedan contribuir a él.

e) El establecimiento de vínculos emocionales e intelectuales entre los miembros para la consecución y/o definición de objetivos compartidos.
Como extensión de la anterior conceptualización, proponemos considerar la cultura de la participación cívica a partir de un tipo de participación en el que los objetivos centrales de las personas/comunidades están orientados a:

a) Una búsqueda libre, diversa, crítica e igualitaria, que permite definir una orientación política frente a problemas o temas socioculturales que motivan 0 agrupan a una comunidad.

b) La constitución como parte de un repertorio para la definición de una identidad compartida que recupera de la cultura popular y mediática referentes para crear y otorgar sentido a las acciones y discursos.

La cultura de la participación ha permitido que el debate sobre el consumo y la producción retome preguntas sobre cuáles son las verdaderas capacidades de las personas para efectuar su agencia y cómo es que sus prácticas implican efectos que van más allá de sí mismos 0 los contextos locales en los que se desarrollan. Esta discusión debe analizarse de manera crítica, especialmente frente a la desmedida influencia de las empresas tecnológicas, que a través del capitalismo de plataformas (Srnicek 2017) plantean incertidumbres sobre la libertad y agencia de las personas.

Lo anterior es especialmente relevante ante los retos devenidos por la excesiva vigilancia (Van Dijck, Poell y De Waal 2018, Zuboff 2019) tanto de los Estados como de las empresas, y en las lógicas del colonialismo de datos (Mejías y Couldry 2019) que pone en cuestión cómo se reproducen las lógicas colonialistas en zonas y territorios del mundo que históricamente han sido vulnerables por actores internacionales a través de la extracción de valor y la legitimación de la explotación (Corbel, Newman y Farrell 2022). En este sentido, la herencia del pensamiento marxista sigue presente y se advierte necesaria como una respuesta crítica al sistema capitalista dependiente de las lógicas extractivas, principalmente de los conglomerados mediáticos y tecnológicos. Autores como Fuchs (2017), Srnicek (2017) y Zuboff (2019) han planteado críticas actuales y relevantes para comprender cómo funcionan los sistemas tecnológicos y cuáles son los desafíos que enfrentamos ante la excesiva influencia de conglomerados que privilegian una lógica extractiva, colonialista y basada en el consumo desmedido.

\section{Comunidades de práctica: participación colectiva y comunitaria}

Las comunidades de práctica son un conjunto de personas que se agrupan a partir de intereses, problemas, pasiones u objetivos a través de los cuales profundizan su conocimiento sobre un área de interés en común. Este tipo de grupos sociales suelen ser informales y se encargan de circular información entre sus miembros para homologar o equiparar sus conocimientos, experiencias e intereses y con esto aspirar a que las comunidades perduren (Wenger, McDermotty y Snyder 2002). 


\section{artnodes}

https://artnodes.uoc.edu

Cultura de la participación cívica: movilización transmedia y hacktivismo en comunidades de práctica (casos de fans y makers)

Desde esta perspectiva se hacen evidentes tres conceptos relevantes para evidenciar formas de colaboración y comunicación, en su sentido primigenio de ponerse en común, a saber, la identidad, el poder y la democratización (Aubert, García y Racionero 2009). Estas tres nociones ayudan a reconocer que en este tipo de comunidades lo más relevante es el conjunto de prácticas, es decir, de acciones (individuales 0 grupales) que les dan sentido y les mantienen juntos.

Apelar a las comunidades de práctica como concepto teórico implica reconocerlas como organizaciones sociales que no dependen necesariamente de una lógica estructural jerárquica, sino de una relacional. Esto significa que los grupos no se entienden en función de las formas de autoridad o del ejercicio del poder, sino a partir de las motivaciones, conocimientos, afinidades e intereses de sus participantes, lo cual ocurre a través del sentido que otorgan estos a las acciones y objetivos que desarrollan (Wenger 2001); aunque, por supuesto, esto no es así siempre, dado que los grupos sociales tienden a comportarse de formas inesperadas.

Seguimos la propuesta de Wenger, McDermotty y Snyder (2002) al identificar tres aspectos fundamentales que pueden caracterizar a una comunidad de práctica. El dominio, el cual se trata del conjunto de temas y objetos de interés de la comunidad, que se refiere a los recursos humanos que conforman una red social dinámica que se define por las prácticas, las cuales se entienden como las maneras y procedimientos definidos socialmente para hacer las cosas.

\section{Apuesta etnográfica por las prácticas presenciales y virtuales}

En este apartado se describen los argumentos relevantes en el orden de lo metodológico, y se ofrece también una descripción general comparativa de las comunidades investigadas con el objetivo de conocer el contexto y las circunstancias en las que se originan sus prácticas y actividades.

El planteamiento, reflexiones y resultados presentados en este documento forman parte de una investigación en la que el autor se preguntó sobre las tipologías de la participación de dos comunidades de práctica: fans y makers. El corpus analizado resultó de la realización de una etnografía de las prácticas de las comunidades a través de la implementación de un diseño de herramientas metodológicas que incluyeron la observación virtual y presencial, la realización de entrevistas en profundidad, y la formulación de categorías de análisis a partir de la sistematización de códigos a través del uso del software computacional Atlas.ti, todo lo anterior bajo los postulados de la teoría fundamentada (Strauss y Corbin 2002). En la siguiente tabla se presenta el esquema analítico mediante el cual se siguió el proceso de categorización de las prácticas de participación de las comunidades investigadas.

\begin{tabular}{|c|c|c|c|}
\hline CONCEPTO & DIMENSIÓN & COMPONENTES & OBSERVABLES \\
\hline & Histórica & $\begin{array}{c}\text { Lo mediático / } \\
\text { tecnológico } \\
\text { Lo institucional / } \\
\text { económico }\end{array}$ & $\begin{array}{c}\text { Prácticas visibles en las interacciones con las interfaces } \\
\text { y sistemas comunicativos temporalmente. }\end{array}$ \\
\cline { 2 - 4 } $\begin{array}{c}\text { PRÁCTICAS } \\
\text { DE PARTICIPACIÓN }\end{array}$ & Estructural & $\begin{array}{c}\text { Prácas de consumo, venta, mercado, apropiación, } \\
\text { público-privado y ética hacker-fan. }\end{array}$ \\
\cline { 2 - 4 } & Comunitaria & $\begin{array}{c}\text { Lo organizacional / } \\
\text { operativo }\end{array}$ & $\begin{array}{c}\text { Prácticas de colaboración, afiliación, trabajo en red, } \\
\text { negociación, liderazgos, reglas y valores. }\end{array}$ \\
\cline { 2 - 4 } & Individual & $\begin{array}{c}\text { La trayectoria / } \\
\text { lo cognitivo-experiencial }\end{array}$ & $\begin{array}{c}\text { Prácticas de significación, expresión, ocio, formación, } \\
\text { aspiraciones, emociones y sentimientos. }\end{array}$ \\
\hline
\end{tabular}

Tabla 1. Esquema analítico de las prácticas de participación

Fuente: elaboración propia

Se utilizó la propuesta de Charmaz (2001) para formular preguntas para facilitar el proceso de codificación. Tales preguntas fueron: ¿Qué está sucediendo?, ¿qué está haciendo la gente?, ¿qué está diciendo el sujeto?, ¿qué dan por supuesto esas acciones y declaraciones?, y ¿de qué manera la estructura y el contexto sirven para comprender esas acciones y declaraciones?

El proceso de codificación supuso un ejercicio detallado de formular preguntas a los datos, esto a través del reconocimiento de que los códigos tienen su origen tanto en los referentes teóricos como en los datos empíricos analizados. Este proceso relacional de establecer vínculos entre sus propiedades y dimensiones similares se llama codificación axial (Gibbs 2007), y permitió la generación de códigos y relaciones entre estos, así como generación de categorías y su jerarquización para configurar un modelo analítico de datos empíricos que combinan el método etnográfico en la recolección de datos y la teoría fundamentada para su sistematización y análisis.

Como se observa en la ilustración siguiente, el proceso de categorización estuvo basado en los textos escritos resultantes de las entrevistas realizadas, como en los textos visuales resultantes del proceso de observación virtual, lo que permitió incluir la riqueza de las prácticas discursivas de la participación que ocurren a través de plataformas sociodigitales. En este ejemplo se observa un post y los códigos que se le asocian en función del contenido compartido y de las interacciones que suscitó. 


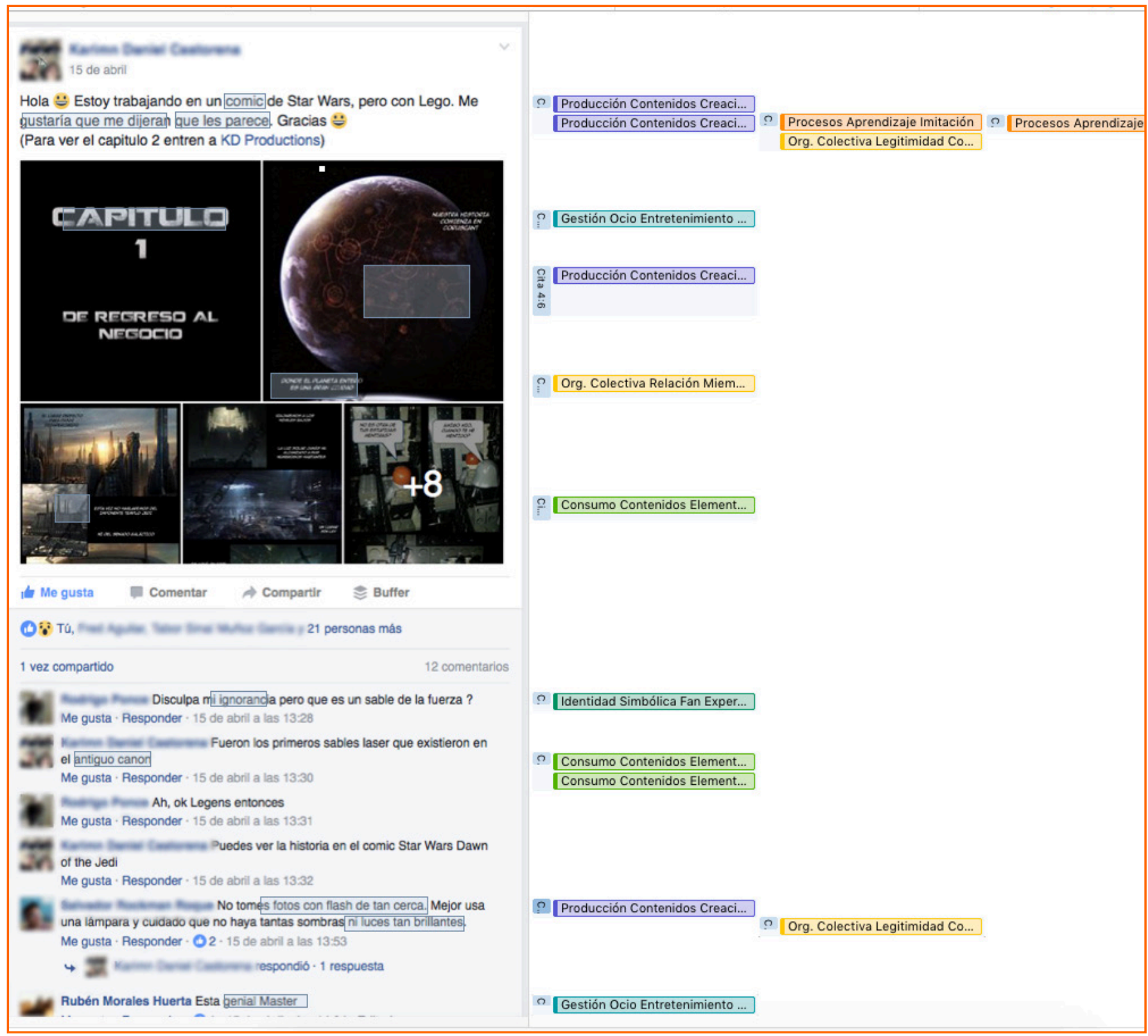

Figura 1. Codificación de imágenes en Atlas.ti

Fuente: elaboración propia

Uno de los principales aspectos de la investigación es que ambos tipos de grupos sociales son equiparables, porque más allá de sus características, contextos, identidades, finalidades e intereses ambos se organizan (y responden) en torno a discursos mediáticos específicos que visibilizan sus pasiones e intereses, tanto individuales como colectivos. Esto resultó a partir de la observación constante de sus formas de agencia comunicativa y organizacional concretadas a través del uso de plataformas y espacios sociodigitales, y mediante la planificación y realización de reuniones presenciales periódicas para discutir, mostrar, enseñar, aprender y disfrutar de sus intereses.

En la tabla 2 se presenta un resumen comparativo de la estrategia metodológica y las fases para su implementación y desarrollo. 


\begin{tabular}{|c|l|c|c|}
\hline \multirow{2}{*}{$\begin{array}{c}\text { FASES DEL PROCESO } \\
\text { DE INVESTIGACION }\end{array}$} & \multicolumn{1}{|c|}{ TIPO DE REGISTRO OBTENIDO PARA EL ANÁLISIS } & \multicolumn{2}{|c|}{ PERSONAS DE LAS COMUNIDADES INVOLUCRADAS } \\
\cline { 3 - 4 } & \multicolumn{2}{|c|}{ EN EL PROCESO } \\
\hline DOCUMENTAL & Recuperación conceptual & 0 & 0 \\
\hline EXPLORATORIA & Notas de campo y fotografías & 3403 & 1358 \\
\hline $\begin{array}{c}\text { OBSERVACIÓN VIRTUAL } \\
\text { OBSERVACIÓN PRESENCIAL }\end{array}$ & $\begin{array}{l}\text { Notas de campo, capturas de pantalla, registros } \\
\text { visuales, audiovisuales, sonoros, y textuales }\end{array}$ & 85 & 42 \\
\hline $\begin{array}{c}\text { ENTREVISTAS EN PROFUNDIDAD } \\
\text { VIRTUALES }\end{array}$ & Regas de campo y fotografías & $33-38$ & $15-20$ \\
\hline $\begin{array}{c}\text { ENTREVISTAS EN PROFUNDIDAD } \\
\text { PRESENCIALES }\end{array}$ & Registros sonoros y textuales & 6 & 7 \\
\hline CODIFICACIÓN & Codificación manual a través de Atlas.ti & 6 & 4 \\
\hline CATEGORIZACIÓN & Categorización manual a través de Atlas.ti & - & - \\
\hline
\end{tabular}

Tabla 2. Estrategia y fases de la investigación

Fuente: elaboración propia

Como se puede observar, además, la estrategia metodológica diseñada implicó una aportación a los debates (Estalella y Ardèvol 2007) sobre los límites de la etnografía como enfoque epistémico que plantea nuevas preguntas sobre cómo asumir la noción de acceso, campo, observación, registro, informante, y entrevista, en las investigaciones que se preguntan por las formas de agencia de las personas más allá de límites tradicionales de espacio/tiempo. En este sentido, creemos que esta experiencia metodológica puede ser de utilidad para la investigación orientada a las comunidades de práctica.

Metodológicamente, este trabajo aporta una estrategia que combina el estudio de espacios y dinámicas que ocurren online y offline, lo cual impulsó el desarrollo de una estrategia de construcción de datos a medida, a través de la observación participante en ambas modalidades y la ejecución de entrevistas en profundidad a informantes clave. Para lo cual fue necesario desplegar un aparato interpretativo capaz de mediar la variedad de los datos reconstruidos y la configuración espacio-temporal de las experiencias. En este sentido, la estrategia desarrollada motivó una constante revisión epistemológica sobre las dimensiones éticas, para lo cual se tomó como referencia las dificultades éticas descritas en la literatura (Estalella y Ardèvol 2007), y se privilegió la perspectiva de los sujetos de investigación, así como las expectativas de la privacidad. En este sentido, no se decidió el carácter privado o público a priori o solo basado en las disposiciones técnicas (de software, por ejemplo), sino, a partir de un ejercicio situacional y dialógico se permitió encontrar el sentido que los individuos conceden a un determinado contexto y contenido. El conocimiento y la comprensión de la perspectiva de los sujetos investigados fueron clave para armar una ética que tomó en cuenta las propiedades del contexto y las elecciones de las personas sobre su agencia y discursos. ${ }^{5}$

En la tabla 3 se presenta un ejercicio comparativo de ambas comunidades con la finalidad de mostrar algunas de sus características, contextos y puntos de encuentro. También se podría considerar como una tabla que ofrece un panorama general a algunos de los aspectos semejantes más relevantes de cada comunidad, y cómo es que sus prácticas son similares entre sí, y las formas de participación que se desarrollan según los temas o causas que motivan las formas de participación cívica.

Si bien es amplio el conocimiento sobre las prácticas que desarrollan tanto las comunidades de fans como las comunidades makers, es importante describirlas especialmente a partir de los temas $y / 0$ causas que motivan sus formas de participación hacktivista. Esto es, mientras que los fans de Star Wars, orientan sus actividades en la construcción identitaria y al altruismo dirigido a grupos vulnerables, los makers buscan sumarse a causas colectivas de naturaleza mucho más internacional como el movimiento OpenSource, o causas basadas en la libertad de internet y en favor de la neutralidad de la web.

5. Desde la dimensión ética, todos los sujetos participantes, de las fases de observación presencial y entrevistas tuvieron pleno conocimiento y aceptaron formar parte de este estudio. 


\begin{tabular}{|c|c|c|}
\hline NOMBRE & Star Wars Fan Club Guadalajara & Makers GDL \\
\hline AÑO DE INICIO & 1999 & 2014 \\
\hline $\begin{array}{l}\text { NARRATIVA MEDIÁTICA } \\
\text { QUE LES AGRUPA }\end{array}$ & $\begin{array}{l}\text { Narrativa transmedia ficcional sobre Star } \\
\text { Wars }\end{array}$ & $\begin{array}{l}\text { Discursos mediáticos no ficcionales sobre } \\
\text { la cultura Makery el Do It Yourself }\end{array}$ \\
\hline N. ${ }^{\circ}$ DE MIEMBROS VIRTUALES & 4.520 & 4.100 \\
\hline $\begin{array}{l}\text { N. }{ }^{\circ} \text { DE MIEMBROS PRESENCIALES } \\
\text { (RECURRENTES) }\end{array}$ & 33-38 & $15-20$ \\
\hline $\begin{array}{l}\text { N.. DE MIEMBROS LÍDERES MÁS } \\
\text { ANTIGUOS EN EL GRUPO }\end{array}$ & $7-9$ & $8-12$ \\
\hline $\begin{array}{l}\text { EDAD PROMEDIO DE LOS } \\
\text { MIEMBROS }\end{array}$ & 33 & 24 \\
\hline $\begin{array}{l}\text { LUGARES DESDE DONDE SE } \\
\text { PARTICIPA }\end{array}$ & $\begin{array}{l}\text { Guadalajara, CDMX, Monterrey, Veracruz, } \\
\text { Los Ángeles (EUA), Puebla, Puerto Vallarta, } \\
\text { Tijuana. }\end{array}$ & $\begin{array}{l}\text { Guadalajara, Monterrey, CDMX, Nueva York, } \\
\text { San Francisco (EUA). }\end{array}$ \\
\hline $\begin{array}{l}\text { REDES Y PLATAFORMAS } \\
\text { COMUNICATIVAS/ } \\
\text { ORGANIZACIONALES } \\
\text { PREDOMINANTES }\end{array}$ & $\begin{array}{l}\text { Facebook, WhatsApp, Messenger (Facebook), } \\
\text { Instagram, sitio web. }\end{array}$ & $\begin{array}{l}\text { Slack, Facebook, Twitter, Trello, YouTube, Sitio } \\
\text { Web. }\end{array}$ \\
\hline $\begin{array}{l}\text { TIPOS DE ENCUENTROS } \\
\text { Y ACTIVIDADES PRESENCIALES }\end{array}$ & Juntas, foros, celebraciones y marchas. & $\begin{array}{l}\text { Fiestas, pláticas, trabajo en red, ferias y } \\
\text { conferencias. }\end{array}$ \\
\hline $\begin{array}{l}\text { TIPOS DE PRODUCCIÓN } \\
\text { MEDIÁTICA DIGITAL }\end{array}$ & $\begin{array}{l}\text { Memes, entrevistas, ilustraciones, } \\
\text { colecciones, memorias, recopilaciones y } \\
\text { reseñas. }\end{array}$ & $\begin{array}{l}\text { Videos tutoriales, infográficos, manuales, GIF, } \\
\text { memes y reseñas. }\end{array}$ \\
\hline $\begin{array}{l}\text { TIPOS DE PRODUCCIÓN } \\
\text { DE OBJETOS O TECNOLOGÍA }\end{array}$ & $\begin{array}{l}\text { Trajes/disfraces, juguetes, objetos de la } \\
\text { narrativa (armas), pinturas y libros. }\end{array}$ & $\begin{array}{l}\text { Código de programación, carpintería/ } \\
\text { muebles, robótica, herramientas, metalurgia, } \\
\text { componentes electrónicos etc. }\end{array}$ \\
\hline $\begin{array}{l}\text { TEMAS O CAUSAS } \\
\text { PARA LA PARTICIPACION CIVICA }\end{array}$ & $\begin{array}{l}\text { - } \quad \text { Conciencia sobre el autismo y asperger. } \\
\text { - } \text { Apoyo a Nariz Roja (organización en pro } \\
\text { - Donañez con cáncer). } \\
\text { - Entrega de jangre. } \\
\text { - Clases y talleres a la niñez. }\end{array}$ & $\begin{array}{l}\text { - Movimiento OpenSource. } \\
\text { - A favor del copylefty licencias abiertas. } \\
\text { - Movimiento anticensura en internet. } \\
\text { - En favor de la neutralidad de la red. } \\
\text { - Contra el consumismo desmedido. } \\
\text { - Formación en uso de tecnología. }\end{array}$ \\
\hline
\end{tabular}

Tabla 3. Características generales y aspectos comparativos de las comunidades Fuente: elaboración propia

\section{Resultados}

\subsection{Movilización transmedia a través del hacktivismo y el altruismo}

Las prácticas de participación de las comunidades de práctica tienen implicaciones cívicas y políticas visibles en formas de hacktivismos y altruismos. La dimensión comunicativa y mediática es pieza clave para ligar el posicionamiento político/cívico de los participantes con la gene- ración de experiencias relevantes como resultado de sus prácticas que transitan por la apropiación (la curación, el remix, la recirculación, la programación y la producción de contenidos/experiencias/objetos) que inciden de forma concreta, directa y física en causas y problemáticas sociales ancladas localmente, pero con referencias globales. Al usar el concepto movilización transmedia (Costanza-Chock 2010), se busca problematizar la relación entre la cultura participativa, la dimensión cívica de esa participación y los referentes mediáticos que la hacen posible 0 que la influyen. 


\section{artnodes}

https://artnodes.uoc.edu

Cultura de la participación cívica: movilización transmedia y hacktivismo en comunidades de práctica (casos de fans y makers)

En este sentido, la movilización es transmedia porque:

a) Se usan elementos narrativos ficcionales, diversidad de medios y lenguajes para comunicar y evocar emociones en pro de una causa o tema concreto.

b) La participación de las personas expande los significados mediáticos y permite la creación colectiva de los miembros.

c) Facilita que las problemáticas sociales de interés para las comunidades se resuelvan a través de referentes mediáticos que evocan el interés a través de las emociones.

Estas formas de movilización tienen dos variantes: la primera es el hacktivismo (Coleman 2014), desarrollado por ambas comunidades, basado en la ética hacker (Himanen 2011), desde la cual se busca incidir socialmente a partir de la defensa de los derechos digitales, el derecho al olvido, a la privacidad y a una red neutral, etc.; y también en la búsqueda de un reconocimiento que haga visible la importancia del acceso a las herramientas de construcción y fabricación para democratizar la invención y la producción de valor incidiendo en las cadenas productivas establecidas.

El hacktivismo observado en esta investigación se materializó a través de la dimensión educativa en términos de los accesos, las jerarquías, las producciones y las formas de validación de la información. Como se ha documentado en diversas ocasiones (Burgos Pino 2014, Sorell 2015, Fitri 2020), el hacktivismo se entiende como una modalidad del activismo, en el que el uso de la tecnología es determinante para las acciones de resistencia, protesta 0 acción política (López Rod, Martín y Martínez 2020). Esta modalidad de activismo no depende solamente de las tecnologías digitales, sino del compromiso social y de la capacidad para fomentar la colaboración en red, para lo cual los usos alternativos de las redes sociodigitales y plataformas tecnológicas es fundamental. ${ }^{6}$ Sus efectos se observan en la vida pública (a través de la diversificación de la participación), a partir de la transformación de los accesos y producción de la información y en la conformación de comunidades, y como base de movimientos sociales (al facilitar el ensamblamiento de intereses, voluntades y pasiones). Lo anterior es relevante también en términos educativos, especialmente en la dimensión del aprendizaje para toda la vida (Aubert, García y Racionero 2009), o de aquellos que ocurren fuera de las instituciones escolares. ${ }^{7}$

La segunda dimensión de la movilización transmedia es el altruismo ficcional, que se basa en la concreción de vínculos sociales para el establecimiento y la participación en redes de apoyo y colaboración en pro de causas sociales o grupos vulnerables. Lo narrativo y ficcional de este tipo de altruismo se utiliza para propiciar un entorno de confianza que facilite el acceso a una participación y cooperación plena y signi- ficativa. Ambas dimensiones, tanto el hacktivismo como el altruismo ficcional, son dos categorías que surgieron del análisis de las comunidades investigadas y de las diferencias entre ellas, especialmente en cuanto a su capacidad de agencia comunicativa y organizacional, así como en los temas que las convocan.

La movilización transmedia considera los tipos y niveles de la participación, sobre todo en términos generacionales, estéticos, educativos y políticos, porque permite conocer las disposiciones, actitudes y valores de la alfabetización tecnológica que despliegan en cada comunidad y a partir de cada tipo de discurso mediático ficcional.

Lo anterior es evidente en ambas comunidades porque los fans de la cultura maker buscan constantemente puntos de entrada y salida para diversificar sus formas de producción y comunicación; y por su parte, los fans se atreven paulatinamente a proponer nuevos usos de las redes sociales y a la producción alternativa de obras mediáticas y piezas físicas basadas en relatos ficcionales con la motivación de ser más efectivos en sus estrategias de apoyo a grupos vulnerables. Esto se pudo observar reiteradamente, sobre todo cuando tanto fans como makers participaron en eventos especiales organizados por ellos o por otras comunidades, como las marchas por la comunidad LGBT, el apoyo a las personas del espectro asperger 0 el llamado a la neutralidad de la red.

Una forma de diferenciar a las comunidades investigadas se puede hacer a partir de las herramientas que usan para producir los objetos 0 discursos de su interés. Los miembros de la comunidad maker constantemente están experimentando nuevas formas de producción y de comunicar sus resultados y experiencias. De esta manera, la diferencia generacional se acentúa porque los fans no muestran el mismo desarrollo de las habilidades y los valores que apuesten por la experimentación, investigación y transformación de los usos y significados establecidos. Otra causa resultante de esta diferencia radica también en los perfiles de los miembros de cada comunidad; mientras en el caso de la comunidad de fans de Star Wars (SW) los miembros son menos especializados y con una orientación más artística o humanista, los makers provienen de formaciones y carreras universitarias orientadas a la ingeniería o a profesiones STEAM ${ }^{8}$ (Honey y Kanter 2013).

Como se puede observar en la tabla 3, en el criterio relativo a las causas o temas para la participación cívica, en ambas comunidades hay un interés por involucrarse en asuntos problemáticos 0 urgentes que requieren de la ciudadanía para ser resueltos. Participan en causas que se pueden agrupar en dos grandes categorías: en una forma de altruismo que hace uso de los referentes mediáticos para ser de fácil acceso y crear empatía hacía el exterior de las comunidades, y, por otro lado, una forma de hacktivismo que concentra todos sus esfuerzos en

6. Diversos enfoques han desarrollado el hacktivismo, ya sea desde una perspectiva artística (Fernández Castrillo 2021), comunicativa (Torres-Soriano, 2018), política (Burgos Pino 2014), de movimientos sociales (Lechón y Mena 2019) 0 compromiso social (García-Estévez 2018).

7. Si bien la dimensión educativa de las prácticas de participación fue central en la pregunta de investigación que da origen a este texto, aquí el enfoque está en las prácticas de participación orientadas al hacktivismo.

8. Expresión en inglés que significa: ciencia, tecnología, ingeniería, artes y matemáticas. 


\section{artnodes}

https://artnodes.uoc.edu

Cultura de la participación cívica: movilización transmedia y hacktivismo en comunidades de práctica (casos de fans y makers)

la resolución de problemas agudizados por el uso excesivo (o poco crítico) de la tecnológica digital interactiva. A saber, altruismo y hacktivismo son dos caras de una misma moneda. En el siguiente apartado se desarrolla cómo es que el hacktivismo se produce a través de acciones comunicativas y de la implementación de vínculos con grupos sociales externos a la comunidad.

\subsection{Las comunidades de fans y makers: estudios de caso de la cultura participativa}

Las comunidades de práctica investigadas constantemente dedican sus actividades a crear y mantener la confianza y credibilidad para desarrollar una voz que las posicione en cierto lugar de legitimidad basada en el conocimiento, relaciones e inteligencia colectiva (Rheingold 2002, Atlee 2003, Lévy 2004), para ser escuchados y tener influencia en otros. Esto ayuda a transformar su realidad social a partir de la empatía y la capacidad para comprender las condiciones de vida de otras personas que no tienen acceso a la tecnología, a la información y no pueden entregarse al intelecto, a la reflexión, a las emociones y a la construcción de un modo y estilo de vida basado en las actividades y temáticas que encausan su pasión (cualquiera que esta sea).

A través del hacktivismo y el altruismo, la movilización transmedia de las comunidades implica formas para representar los deseos de un lugar/momento mejor, y una constante para hacer notar sus propias posturas políticas, ideológicas, educativas y de valores que dan sentido a sus prácticas cotidianas. Uno de los fundadores de la comunidad de Star Wars lo pone en los siguientes términos: «colaborar con comunidades y organizaciones civiles sirve para sacar las propias preocupaciones [...] desde que nos han invitado, motivamos a que los miembros aprendan el valor de otras cosas más allá de SW» (Carla, S. ${ }^{9}$ Comunicación personal, 21 de agosto de 2019). Reflexión que permite conectar las prácticas colaborativas de la comunidad con las actividades que otros grupos y asociaciones realizan como parte de sus actividades cotidianas en beneficio de causas altruistas y cooperativas en pro de asumir su responsabilidad individual y grupal con la sociedad.

El hacktivismo observado sucedió mediante el uso y la apropiación de algoritmos, códigos y lenguajes informáticos, pero especialmente a partir de acciones sociales y culturales compartidas en búsqueda de una transformación o mejoramiento de la sociedad. En ambas comunidades se observaron prácticas de participación tanto hacktivista como de naturaleza altruista. De hecho, creemos que estas dos dimensiones de su agencia ocurren como parte de un mismo impulso colectivo para lograr relevancia social y para dar salida a sus preocupaciones o temas de interés. Desde esta perspectiva es importante asumir que una parte esencial de las motivaciones a la participación se ubican en el ámbito de lo lúdico y del ocio que implica entregarse a temas que no necesariamente se traducen en un beneficio económico, pero sí en una forma de disfrute compartido, que directa 0 indirectamente reconoce la importancia de vivir en sociedad y de aportar alguna solución a los problemas de la sociedad desde una mirada de corresponsabilidad.

Como se puede observar en la tabla 4, las diferencias entre las comunidades investigadas en materia de hacktivismos y altruismos se dan especialmente a los temas de su interés y en cómo dichos intereses se traducen en acciones y discursos que ayudan al propio funcionamiento de cada comunidad de práctica y que además busca aportar al exterior de estas.

Como se puede observar, una diferencia notable es el hecho de que Ios fans de SW desarrollan más prácticas desde la perspectiva altruista, mientras que la comunidad de makers lo hace desde el hacktivismo. Esto se puede explicar tanto por la formación de sus miembros, como por las diferencias generacionales o incluso por la manera en que deciden participar en la sociedad.

En el caso de los fans, sumarse a causas altruistas es una forma de aspirar a la empatía y comprensión del otro (diferente-distinto), que en la pantalla-ficción (y discursos mediáticos) se representa poco, pero que los fans interpretan en una doble vía: ya sea como algo ausente necesario de representar, o como una reflexión implícita en la lucha constante entre el bien y el mal mostrado en las narrativas que consumen.

En el discurso usado y en las acciones recurrentes realizadas por los makers, constantemente queda reflejada una crítica al sistema educativo del que ellos formaron parte (y que muchas veces replican involuntariamente), con el objetivo de hacer evidente a la opinión pública (y para ellos mismos) que hay saberes y experiencias de aprendizaje que (según ellos) no se pueden construir en la escuela.

Esta idea es una forma de posicionamiento político-ideológico que busca mantener a la comunidad al margen de las estructuras de poder institucional, para seguir siendo diferentes y marginados. Y, además, se puede interpretar en sí misma como una acción hacktivista toda vez que busca la transformación social a través del uso crítico de tecnologías.

Las estrategias para realizar lo anterior tienen un componente comunicacional basado en la apropiación tecnológica, la propuesta de nuevas configuraciones a partir de la creación de software e interfaces que sean capaces de evidenciar las fallas de las metodologías tradicionales para aprender, transmitir información y validar el conocimiento. Y a partir de la experimentación constante en el uso de plataformas y sistemas, que amplíen su rango de influencia y redimensionen su voz y mirada.

9. Todos los nombres de las personas entrevistadas son seudónimos 


\section{artnodes}

\begin{tabular}{|c|c|c|}
\hline & HACKTIVISMOS & ALTRUISMOS \\
\hline FANS DE SW & $\begin{array}{l}\text { Distribución digital de materiales y contenidos } \\
\text { de la narrativa de SW. } \\
\text { - Creación de repositorios y bases de datos de } \\
\text { contenidos y productos mediáticos relacionados } \\
\text { con la ficción y otros temas actuales. } \\
\text { - Acciones de regulación del mercado de piezas y } \\
\text { productos coleccionables. }\end{array}$ & $\begin{array}{l}\text { - Entrega de regalos a niños enfermos. } \\
\text { - } \text { - Acompañas de donación sanguínea. } \\
\text { asperger. } \\
\text { - Eventos y caminatas en pro de los derechos de } \\
\text { las infancias. } \\
\text { - Recolección y entrega de materiales escolares. } \\
\text { - Invitación a funciones de SW a personas } \\
\text { diversas. }\end{array}$ \\
\hline MAKERS & $\begin{array}{l}\text { - Impulso a la reparación de equipos de cómputo. } \\
\text { - } \text { Campañas para observar y mejorar la movilidad } \\
\text { - Impulso al uso e implementación de acciones } \\
\text { ecologistas como la creación de composta y el } \\
\text { reciclaje casero. } \\
\text { - Aportaciones técnicas, basadas en el análisis } \\
\text { de datos, para incentivar el uso de vehículos no } \\
\text { motorizados. } \\
\text { - Creación de objetos a través de tecnologías de } \\
\text { fácil acceso. }\end{array}$ & $\begin{array}{l}\text { - Clases de ciencias y matemáticas a niños. } \\
\text { - Apertura de espacios físicos para que otras } \\
\text { comunidades ofrezcan actividades educativas o } \\
\text { lúdicas. }\end{array}$ \\
\hline
\end{tabular}

Tabla 4. Tipos de hacktivismos y altruismos desarrollados por las comunidades

Fuente: elaboración propia

...............

\section{Conclusiones: cultura de la participación cívica a través del hacktivismo}

En el contexto de la cultura participativa, a través de las dinámicas transmediales de la comunicación y la cultura, otras categorías surgen como posibilidades para condensar la diversidad de la participación, del compromiso y de la agencia. La imaginación cívica (Jenkins et al. 2016) define la capacidad para vislumbrar alternativas para mejorar el momento actual (y futuro) de las instituciones políticas, educativas, económicas y culturales. Así pues, esta forma de imaginación posibilita la gestión de las esperanzas y pasiones, en tanto que, a través de las prácticas cotidianas, se busca realizar contribuciones que permitan mejorar lo que no funciona, lo que está mal, o debería funcionar mejor (desde la perspectiva de las comunidades).

En el caso de los makers, esta imaginación cívica y gestión de la esperanza, a primera vista, parece que está orientada al uso de la tecnología; pero, más bien, la dimensión tecnológica es una forma de concretar esa imaginación, y el vehículo que permite (muchas veces basado exclusivamente en un sentido lúdico y en una actitud rebelde) probar otras alternativas para hacer las cosas. En este sentido, la imaginación cívica supone redimensionar el hacktivismo, a través de una ética de la participación que tenga efectos materiales, tangibles y observables en la vida pública, en el quehacer político y en la cultura como posibilidades para dejar presencia de su existencia y ganarse su lugar en el mundo.

El hacktivismo de los fans es una forma de movilización transmedia que impulsa un afán democratizador de lo informacional y comunicacional, a través del uso y apropiación de algoritmos, códigos y lenguajes informáticos, pero especialmente a través de las pautas sociales y culturales que normalizan y popularizan prácticas de consumo y sobre todo de producción que ofrezca nuevos sentidos a problemas sociales. Desde esta perspectiva, el hacktivismo de fans es una forma de participación que está moldeando la cultura, mediante el uso y la apropiación de la tecnología tanto digital como de aquella que es analógica.

Una manera de evaluar la efectividad de las prácticas, tanto hacktivistas como altruistas, de fans y makers es el propio hecho de su constante agencia ante los diversos retos que enfrentan o los temas que son de su interés. En este sentido, se puede tomar como referencia el crecimiento constante de ambas comunidades, bien en su dimensión virtual bien en su agencia presencial. Y, además, hay que señalar que su capacidad de influencia social ha logrado establecer vínculos relevantes con otras comunidades e incluso con algunas instancias 


\section{artnodes}

https://artnodes.uoc.edu

Cultura de la participación cívica: movilización transmedia y hacktivismo en comunidades de práctica (casos de fans y makers)

del Gobierno local. ${ }^{10}$ La efectividad de las prácticas de participación debe ser analizada en su contexto, especialmente porque implica una búsqueda de transformación de los entornos personales, familiares y de las propias comunidades. En este sentido, así lo explica un miembro de la comunidad maker. «...trabajar en proyectos es interesante porque es una forma de poner a prueba lo que sabes [...] y lo que puedes aprender al trabajar con tus compañeros, que son especialistas en temas diferentes a los propios» (Lorenzo, R. Comunicación personal, 2 de septiembre de 2019).

De lo anterior se puede desprender también una crítica a la educación tradicional, la cual puede retomar algunos planteamientos de la ética y cultura hacker, especialmente a partir de una autocrítica que haga evidente las cosas que no funcionan a la luz de lo que ha cambiado en la cultura y en la vida de las personas, especialmente para los jóvenes.

Imaginar alternativas de solución a los problemas sociales es una tarea pendiente que se trata de resolver con una constante expectativa de futuro representado en las ficciones, no solo como aspiración, sino como horizonte que delinea hacia dónde es posible ir, para bien o para mal. Ya sea un futuro prometedor y más justo para todos, o porque se convierta en una realidad que no da cabida a formas distintas de ser, decir y hacer las cosas.

Cuando las comunidades de práctica recuperan sus referentes narrativo-mediáticos para elaborar sus propias producciones, están desplegando la imaginación cívica, ya que esto les permite comprometerse con (y reproducir) una idea de mundo y sociedad de la cual son parte. Bajo esta óptica, la vida de la comunidad adquiere un nuevo sentido, si se le ve como una oportunidad para aprender a ser ciudadano a partir de un conjunto de aspiraciones compartidas que suponen un constante ir y venir entre ficción, sociedad, tecnología y actualidad.

Lo anterior propicia el desarrollo de un alfabetismo cívico que visibiliza los problemas de la sociedad a través de las temáticas de interés de las comunidades, lo que permite que los miembros elijan cuáles son las particularidades del tema (que los convoca y agrupa) que les permite conectar con sus pasiones y necesidades personales.

La cultura de la participación y la movilización transmedia, a través del hacktivismo y el altruismo, implican la configuración de espacios y dinámicas compartidas en las que es posible la crítica, el debate y la expresión pública, que, si bien se origina a partir de temáticas específicas, estas tienen influencias y componentes sociales y culturales relevantes para la construcción de espacio y debate público. Hay todavía mucho por investigar sobre este tema y muchas preguntas que formular sobre las culturas de la participación y los diferentes grupos sociales que las practican, especialmente si se consideran las complejas discusiones que ocurren sobre temas fundamentales como la propagación de noticias falsas (Riva-Casas 2019), el colonialismo de datos (Mejías y Couldry 2019), la vigilancia (Zuboff 2019) y el capitalismo de plataformas (Srnicek 2017).

\section{Referencias bibliográficas}

Aparici, Roberto, Osuna-Acedo, Sara. «La Cultura de la Participación». Revista Mediterránea de Comunicación, vol. 4, no. 2 (2013): 137148. DOI: https://doi.org/10.14198/MEDCOM2013.4.2.07.

Atlee, Tom. «ls collective Intelligence like individual Intelligence?». The Co-Intelligence Institute (2003). https://www.co-intelligence.org/ CollectiveAndlndivIntell.html. [Fecha de consulta: 19/01/22].

Aubert, Adriana, García, Carme, Racionero, Sandra. «El aprendizaje dialógico». Cultura y Educación, vol. 21, no. 2 (2009): 129-139. D0l: https://doi.org/10.1174/113564009788345826.

Avogadro, Marisa. «Hacktivism: Entramado invisible del Ciberpoder». Revista Razón y Palabra, no. 77, año. 16 (2011). México: Instituto Tecnológico y de Estudios Superiores de Monterrey. Estado de México.

Burgos Pino, Edixela. «El Hacktivismo entre la participación política y las tácticas de subversión digital». Razón y Palabra, vol. 18 no.88 (2014): 6-26.

Charmaz, Kathy. «Grounded Theory». En: R. Emerson (Ed.). Contemporary Field Research: Perspectives and Formulations (2001): 335352. Prospects Heights, Estados Unidos: Waveland Press.

Coleman, Gabriella. Hacker, hoaxer, whistleblower, spy: the many faces of Anonymous. Londres: Verso, 2014.

Corbel, Christofer, Newman, Trent, Farrell, Lesley. «Gig Expectations: Literacy Practices, Events, and Texts in the Gig Economy». Written Communication 39, no. 1 (2022): 66-96. D0l: https://doi. org/10.1177/07410883211052941.

Costanza-Chock, Sasha. (2010). Se ve, se siente: transmedia mobilization in the Los Angeles immigrant rights movement. Dissertation Doctor of Philosophy (Communication). California: University of Southern California. http://web.mit.edu/schock/www/docs/transmedia-mobilization-scc-diss.pdf.

Couldry, Nick. «More Sociology, More Culture, More Politics: Or, a modest proposal for "convergence" studies». Cultural Studies, vol. 25, no. 4/5 (2011): 487-501. DOl: http://doi.org/10.1080/09502386.2 011.600528

Estalella, Adolfo, Ardèvol, Elisenda. «Ética de campo: hacia una ética situada para la investigación etnográfica de internet». Forum Qualitative Social Research, vol. 8, no. 3 (2007): 1-25. D0l: https://doi. org/10.17169/fqs-8.3.277.

10.En el caso de la comunidad maker, sus prácticas de participación les han permitido establecer vínculos y relaciones constantes con instancias gubernamentales y otras organizaciones de sector tecnológico y de innovación. Mientras que, en el caso de los fans de SW, su influencia se concentra especialmente en organizaciones civiles que buscan de alguna manera apoyar a las múltiples infancias 0 a otras minorías. 
Evans, Elizabeth. «The economics of free: Freemium games, branding and the impatience economy». Convergence, vol. 22, no. 6 (2016): 563-580. D0l: https://doi.org/10.1177/1354856514567052.

Fernández Castrillo, Carolina. «La condición transreal: información expandida y hacktivismo en el Media Art». Artnodes, no. 28 (2021). D0I: https://doi.org/10.7238/a.v0i28.377879.

Fitri, Nofia. «Democracy Discourses through the Internet Communication: Understanding the Hacktivism for the Global Changing». Online Journal of Communication and Media Technologies, vol. 1, no. 2 (2020). DOl: https://doi.org/10.29333/ojcmt/2332.

Fuchs, Christian. Digital Labour and Karl Marx. London:Routledge, 2017. García-Estévez, Noelia. «Origen, evolución y estado actual del activismo digital y su compromiso social. Ciberactivismo, hacktivismo y slacktivismo". Congreso Internacional Move.Net Sobre Movimientos Sociales y TIC (2018): 139-156. https://idus.us.es/handle/11441/70636.

Gibbs, Graham. El análisis de datos cualitativos en Investigación Cualitativa. Coruña: Ediciones Morata, 2007.

Hamari, Juho, Hanner, Nicolai, Koivisto, Jonna. «Why pay premium in freemium services? A study on perceived value, continued use and purchase intentions in free-to-play games» International Journal of Information Management, vol. 51 (2020). ISSN 0268-4012. DOI: https://doi.org/10.1016/j.jinfomgt.2019.102040.

Himanen, Pekka. La ética del hacker y el espíritu de la era de la información. (Creative Commons). Barcelona: E-book, 2011.

Honey, Margaret, Kanter, David E (eds.). Design, make, play: Growing the next generation of STEM innovators. Nueva York: Routledge, 2013. DOl: https://doi.org/10.4324/9780203108352.

Jenkins, Henry. Convergence Culture. La cultura de la convergencia de los medios de comunicación. Barcelona: Paidós, 2008.

Jenkins, Henry (2009). Confronting the Challenges of Participatory Culture: Media Education for the 21 Century. Massachusetts: MacArthur-MIT Press. DOI: https://doi.org/10.7551/mitpress/8435.001.0001.

Jenkins, Henry. «Rethinking Convergence/Culture». Cultural Studies, no. 28 (2014, October): 267-297. DOl: https://doi.org/10.1080/095023 86.2013.801579.

Jenkins, Henry, Shresthova, Sangita, Gamber-Thompson, Liana, Kligler-Vilenchik, Neta and Zimmerman, Arely. M. By any media necessary: the new youth activism. Nueva York: New York University Press, 2016.
Lechón Gómez, Domingo M., Mena Farrera, Ramón A. «El hacktivismo e Internet como territorio en disputa. Una mirada desde los marcos de acción colectiva». Estudios Políticos, no.48 (2019). D0l: https:// doi.org/10.22201/fcpys.24484903e.2019.48.70423.

Lévy, Pierre. Inteligencia Colectiva por una antropología del ciberespacio. Washington: Organización Panamericana de la Salud, 2004.

López Rod, A. A., Martín, Á C., Martínez, P. C. «Hacktivism and political participation: Between the criminalization of the unconventional and the constitution of new political actors». RISTI - Revista Iberica de Sistemas e Tecnologias de Informacao, no. E35 (2020): 390-405.

Mejías, Ulises, Couldry, Nick. «Colonialismo de datos: repensando la relación de los datos masivos con el sujeto contemporáneo». Virtualis, vol. 10, no. 18 (2019): 78-97. ISSN 2007-2678.

Rheingold, Howard. Smart Mobs: The Next Social Revolution. NuevaYork: Basic Books, 2002.

Riva-Casas, Andrés. «La alfabetización mediática e informacional en la era del capitalismo de vigilancia». Cuadernos Del CLAEH, vol. 38, no. 110 (2019). D0l: https://doi.org/10.29192/claeh.38.2.15.

Sorell, Tom. «Human rights and hacktivism: The cases of wikileaks and anonymous». Journal of Human Rights Practice, vol. 7, no. 3 (2015): 391-410. DOI: https://doi.org/10.1093/jhuman/huv012

Srnicek, Nick. Platform capitalism. Cambridge: Polity Press, 2017.

Strauss, Anselm, Corbin, Juliet. Bases de la investigación cualitativa: técnicas y procedimientos para desarrollar la teoría fundamentada. Antioquia: Editorial Universidad de Antioquia, 2002.

Torres-Soriano, Manuel. R. «El hacktivismo como estrategia de comunicación». Cuadernos de Estrategia, no. 197 (2018): 197-224. https:// dialnet.unirioja.es/servlet/articulo?codigo $=6518649$.

Van Dijck, José, Poell, Thomas, De Waal, Martijn. Platform society: public values in a connective world. Nueva York: Oxford University Press, 2018. DOl:https://doi.org/10.1093/0so/9780190889760.001.0001. Wenger, Étienne. Comunidades de práctica: aprendizaje, significado e identidad. Buenos Aires: Paidós, 2001.

Wenger, Étienne, McDermott, Richard, Snyder, William. M. Cultivating Communities of Practice. Massachusetts: Harvard, 2002. D0I: http://doi.org/10.1016/j.jchas.2013.03.426.

Zuboff, Shoshana. The age of surveillance capitalism: The fight for a human future at the new frontier of power. Nueva York: Profile Books, 2019. 
CV

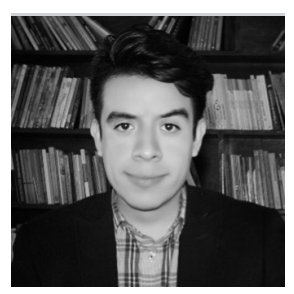

José Manuel Corona-Rodríguez

Instituto Tecnológico y de Estudios Superiores de Monterrey

manuel.corona@tec.mxu

Doctor en Educación y maestro en Comunicación por la Universidad de Guadalajara, México. Licenciado en Ciencias de la Comunicación por la Benemérita Universidad Autónoma de Puebla. Investigador miembro del Sistema Nacional de Investigadores Nivel-C del CONACYT. Profesor visitante en la Facultad de Comunicación de la Universidad Pompeu Fabra en 2017. Coordinador ejecutivo e investigador en la cátedra UNESCO-AMIDI UDG desde 2016. Docente e investigador interesado y con experiencia en: comunicación pública, teorías de la comunicación, alfabetización mediática informacional, narrativas transmedia, estudios de audiencias y cultura digital. Miembro del grupo de investigación internacional del Proyecto Global Kids Online, que investiga el estado actual de los derechos digitales de las infancias en diferentes países del mundo. En dicho proyecto de investigación colaboran, entre otros, Henry Jenkins, Tessa Jolls y Carolyn Wilson. Miembro actual del grupo de investigación encargado del proyecto Análisis de contenido de la representación de personas de la diversidad sexual en contenidos audiovisuales, financiado por el Instituto Federal de Telecomunicaciones.

https://orcid.org/0000-0001-7394-5230

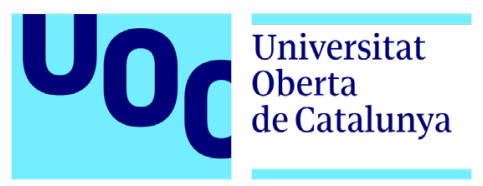

